\title{
Методы инженерной геофизики при поисках техногенных объектов на мелководных акваториях
}

\author{
(C2021 В. С. Стариков ${ }^{\bowtie}$ \\ АО Предприятие подводно-технических работ «Пётр» (Воронеж) \\ ул. Остужева д.2, корп. А, 394042, Воронеж, Российская Федерация
}

\begin{abstract}
Аннотация
Введение: в настоящей работе рассматривается проблема выявления техногенных тел на мелководной акватории скрытых в результате процесса седиментации. В качестве решения задачи предлагается комплексный подход, основанный на результатах интерпретации выполненных измерений модуля вектора магнитной индукции, в соответствии с предлагаемой методикой съемки, поддержанной методами сейсмоакустики.

Методика: в условиях мелководной акватории и ограниченного береговыми линиями пространства исключается использование громоздкого оборудования и крупногабаритных плавательных средств. Отсюда предлагается выработать комплекс методов и методику работ с применением маломерных плавательных средств, при поисках и обнаружении непрогнозируемых дискретных намагниченных объектов. На основе ранее выполненных изысканий на протяженных линейных техногенных объектах была применена пошагово совокупность: гидроакустического, магнитометрического и геоакустического методов. В методическом обеспечении каждого из вида выполняемых работ является разрешимость, которая будет гарантировать выявление целей, и достаточность собранной информации, дающей общее представление о состоянии акватории.

Результаты и обсуждение: гидроакустическое обследование, как средство визуализации, дало представление о степени захламленности дна антропогенными объектами, но не смогло однозначно выявить фрагменты танка, в виду того, что за длительный период времени всё было нивелировано русловыми наносами. В результате выполненных магнитометрических наблюдений были выявлены две значительные аномалии магнитного поля. Произведена количественная интерпретация магнитных аномалий для двухмерного случая: выполнен пересчет в верхнее полупространство, дана оценка глубины заложения объектов. По итогам работы геоакустического метода были уточнены глубины в акватории реки. Совокупные результаты задействованного комплекса позволили обратиться к решению прямой задачи магнитометрии с использованием программной среды Mathcad14. В результате выполненного моделирования в программе Mathcad14 вычисленное поле модуля вектора индукции от совокупности модельных источников, показало хорошую тождественность к наблюдённому полю. Прямые водолазные обследования, которые последовали после завершения стадии изысканий, подтвердили наличие корпуса и башни танка, перекрытых речными наносами. Заключение: основными результатами наших исследований, направленных на обеспечение безопасности хозяйственной деятельности на мелководных акваториях при осуществлении поиска непрогнозируемых намагниченных техногенных объектов, является совершенствование методики гидромагнитной съемки с последующей интерпретацией данных, в том числе с применением компьютерного моделирования, а также выработка оптимального комплекса геофизических работ.

Ключевые слова: мелководная акватория, техногенные объекты, комплекс геофизических методов, магнитные аномалии, моделирование магнитного поля.
\end{abstract}

Контент доступен под лицензией Creative Commons Attribution 4.0 License.

Стариков Вадим Сергеевич, e-mail: stvase@mail.ru 
Для циитирования: Стариков В. С. Методы инженерной геофизики при поисках техногенных объектов на мелководных акваториях// Вестник Воронежского государственного университета. Серия: Геология. 2021. №1. C. 75-81. DOI: https://doi.org/10.17308/geology.2021.1/3339

\section{Введение}

В настоящее время магниторазведка эффективно используется в решении инженерно-геологических и инженерно-технических задач изучения самой верхней части разреза до глубин первые десятки метров с целью выявления техногенных объектов изучаемой среды. При этом, как правило, используются сверхдетальные и высокоточные магнитные измерения [1-4 и др.], фактически отвечающие кондициям микромагнитной съёмки [5]. Магниторазведочные работы такого плана весьма эффективны при изучении протяжённых объектов - трубопроводных систем, с линейными размерами до первых дециметров в поперечнике и залегающих на глубинах до нескольких метров [6-8 и др.]. Реализуемые современные методики высокоточных магнитных исследований при изучении поля от трубопроводных систем, а также развитые алгоритмы интерпретации таких полевых наблюдений [9-12], позволяют достаточно точно определять пространственное положение стальных трубопроводов. Во многом эффективность метода магнитометрии обусловлена относительной простотой модели источника аномалий двухмерной протяжённой сильномагнитной структурой, хотя его общее магнитное поле и осложнено специфическим суммированием аномалий от отдельных элементов трубопровода, как показано в $[12,13]$.

Наряду с этим, применение магнитометрических исследований при поисках и обнаружении непрогнозируемых дискретных намагниченных объектов встречает значительные трудности, что обусловлено влиянием разнородных интенсивных магнитных помех и существенным снижением разрешимости магнитного метода в этих условиях [14-16]. Такое снижение эффективности магнитометрии становится особенно заметным при работах на пресноводных и морских водоёмах, когда данные магниторазведки не могут быть поддержаны георадарными исследованиями [1] в условиях высокой электропроводности водной толщи. Специфика гидромагнитных полевых работ рассматривается в данной работе на примере обнаружения заглублённых относительно дна водоёма дискретных техногенных объектов, представляющих опасность для судоходства и инженерных работ на акваториях [17].

\section{Методика работ}

Геофизические работы на мелководье требуют использования маломерных немагнитных плавательных средств, на которых не всегда возможно устанавливать относительно массивное исследовательское оборудование. В силу этого возрастает роль гидромагнитных наблюдений (включая магнитную градиентометрию) отличающихся высокой производительностью и точностью интерпретации полевых материалов при правильном методическом обеспечении системы наблюдений. Разумеется, только гидромагнитная съёмка, не может решить все задачи инженерных изысканий на акваториях и здесь представляется целесообразным выработать некоторый рациональный комплекс полевых работ, сочетающий преимущества магнитометрического метода и высокую точность других геофизических методов, связанных с поисками объектов техногенного происхождения в условиях пресноводных и морских водоёмов [4, 17]. В качестве такого комплекса предлагается использовать сочетание сейсмоакустических методов (геоакустика и гидроакустика) и собственно гидромагнитную съёмку в различных модификациях. Роль и место каждой из этих съёмок определяется конкретными задачами исследований, спецификой проводимых работ и условиями выполнения полевых геофизических наблюдений.

Важным моментом в методическом обеспечении гидромагнитных работ является надёжная оценка шага съёмки, обеспечивающая гарантированную разрешимость обнаружения элементарных магнитных объектов, залегающих на некоторой максимально возможной глубине. Исследование таких условий обнаружения ранее выполнено для ряда трёхмерных моделей совокупности простых источников $[15,18]$, что является оптимальным для обладающих значительной остаточной намагниченностью, военно-технических артефактов, интенсивно выделяющихся аномальными значениями модуля поля $\Delta B$ (рис.1), в сравнении к более сложному графическому представлению результатов градиентометрической съёмки. Поэтому магнитометрическая разведка проводилась в варианте съёмки модуля вектора индукции магнитного поля $[4,15]$. В частности, для точечных дискретных объектов, залегающих на глубине $H$ и отстоящих друг от друга на расстоянии $\Delta X$, было определено условие гарантированного корреляционного обнаружения каждого отдельного источника в форме $\Delta X \geq 4 / 5 H$. Из последнего соотношения следует строгое требование к шагу гидромагнитных наблюдений, который должен быть $\leq 1 / 5 H$, что является более жёстким ограничением в отличии от известных оценок разрешимости для двухмерного случая [19]. Соблюдение указанного требования обеспечивает достоверное наблюдение магнитных аномалий совокупности объектов и последующую интерпретацию результатов съёмок для каждого из них.

Предложенный комплекс геофизических исследований реализовывался в различных условиях мелководья [4], где, как правило, на первом этапе выполнялись гидромагнитные съёмки, которые позволяют установить самую общую ситуацию на участке работ и наметить площади проведения более детальных наблюдений. В данной работе примером объекта поисков с использованием предложенного комплекса являлся советский танк КВ-1 времён Второй мировой войны, находящийся в судоходной части русла р. Дон. После 
неудачной попытки извлечения танка сразу в послевоенное время, было принято решение об уничтожении данного артефакта взрывом, в результате чего судоходство на тот период было возобновлено. Именно частичное разрушение объекта стало главным осложняющим фактором при определении пространственного положения фрагментов танка по прошествии почти семидесяти лет. Метод гидролокации, применённый на начальном этапе наших поисков, результатов не дал, так как фрагменты объекта оказались за столь длительное время перекрыты русловыми наносами.

На следующей стадии опоискования применялась гидромагнитная съёмка по сети наблюдений с принятыми оптимальными параметрами обнаружения, результаты которой подтвердили наличие двух интенсивных аномалий магнитного поля (рис. 1). Эти аномалии предположительно соответствуют собственно корпусу танка и его бронебашне, отброшенной взрывной волной на расстояние около 40 метров при подрыве машины.

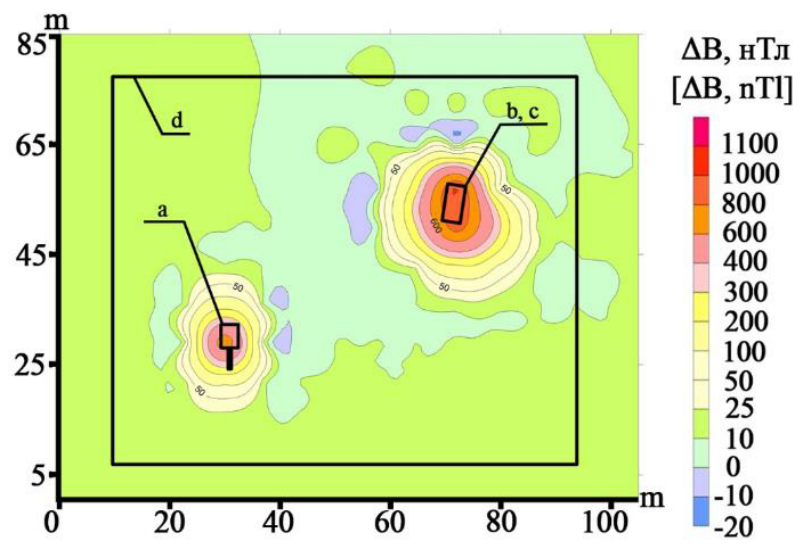

Рис. 1. Аномальное магнитное поле по данным гидромагнитной съёмки русла р. Дон Воронежская область. Пояснение: $a, b, c$ - магнитные объекты; $d$ - область магнитного моделирования

[Fig. 1. Anomalous magnetic field according to the hydromagnetic survey of the riverbed Don, Voronezh Region. Explanation: $a, b, c$ - magnetic objects; $d$ - field of magnetic modeling.]

\section{Интерпретация результатов}

Количественная интерпретация магнитных аномалий выполнялась в двухмерном варианте по методике, изложенной в [20], на основе сопоставления исходного и пересчитанного в верхнее полупространство аномального поля. Оценку глубины залегания точечного объекта $h$ можно вычислить по формуле Левашова, использующей значения максимумов амплитуд исходного $B_{a}(x, 0)_{\max }$ и пересчитанного на высоту $\Delta h$ поля $B_{a}(x, \Delta h)_{\text {max }}$ согласно определению

$$
h=\Delta h \sqrt{B_{a}(x, \Delta h)_{\max }} /\left[\sqrt{B_{a}(x, 0)_{\max }}-\sqrt{B_{a}(x, \Delta h)_{\max }}\right] .
$$

Результаты интерпретации магнитных аномалий по методу [20] и классическими способами характерных точек [5] продемонстрировали сходимость оценок глубины источников поля $h \approx 3.15 \pm 0.20 \mathrm{м}$, что является хорошим примером решения задач инженерной магни- тометрии в условиях мелководной акватории. Высокая точность полученных оценок гарантирована априорными данными о модели источников поля и оптимальным шагом выполненной гидромагнитной съёмки [18].

Полученные оценки глубин положения источников интенсивных магнитных аномалий и известные магнитные свойства современной высококачественной стали $[12,20]$, в предположении, что она примерно отвечает и материалу брони танка, позволили обратиться к решению прямой задачи магнитометрии от составной модели искомого артефакта. Расчёт модуля вектора аномального магнитного поля от составной модели источников выполнялся по известному численному алгоритму адаптивной аппроксимации среды [22], для которого была написана программа расчёта компонент индукции магнитных полей в системе компьютерной математики Mathcad14. Входными параметрами программы являются пространственные характеристики дискретных, произвольно ориентированных в пространстве параллелепипедальных элементов модели среды, которые наделялись магнитными свойствами примерно пропорциональными известным массам фрагментов танка. При расчётах принималось во внимание совокупное влияние индуктивной и остаточной намагниченности материала фрагментов военного артефакта. Полный вектор намагничения каждого объекта принят равным 3 A/м, а ориентировки векторов подбирались в процедуре решения прямой задачи от совокупности объектов. Шаг вычислений поля в прямой задаче магнитометрии отвечал рассмотренному критерию [18] для оптимального обнаружения источников.

На завершающей стадии работ, для оценки глубин дна водоёма применялся геоакустический метод исследований с использованием поддонного профилографа. Район акустического обследования определялся по результатам интерпретации съёмки аномального магнитного поля. Итоговые данные о глубинах дна водоёма, показанные на рис. 2, использовались как верхнее ограничение для оценок положения фрагментов артефакта, при этом принимались во внимание возможные мощности перекрывающих донных отложений (от 0.5 до $1.5 \mathrm{~m}$ ).

Решение прямой задачи магнитометрии от принятой модели было выполнено для фрагмента области наблюдений, включающего основные аномалии наблюдённого магнитного поля. В результате нескольких итераций было рассчитано поле модуля вектора индукции от совокупности модельных источников, пространственное положение которых в упрощённом виде показано на рис. 3. Полученные результаты моделирования демонстрируют: хорошую сходимость диапазонов данных наблюдённого и расчётного полей, а также относительно малые значения разности между измеренным и модельным полем в диапазоне от -15 до +21 нТл (рис. 3). Отметим, что стандартное отклонение невязки полей равно 7.8 нТл, а средняя относительная ошибка аппроксимации подобранной модели составляет 3.9 \%, это позволяет говорить о надёжной оценке 
размеров и глубинного положения основных крупных фрагментов искомого объекта. Данные о положении глубин центров модельных объектов по результатам моделирования магнитного поля установлены как: объект $1-3.3$ м, объект $2-4.5$ м, объект $3-3.5$ м.

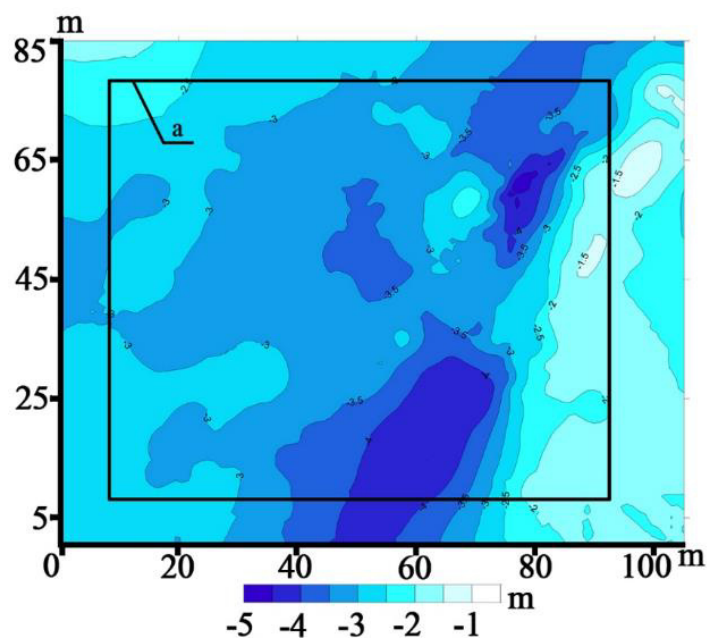

Рис. 2. Карта глубин дна русла р. Дон на участке обследования. Пояснение: а - область магнитного моделирования.

[Fig. 2. Map of the depth of the bottom of the riverbed Don at the survey site. Explanation: a - field of magnetic modeling.]

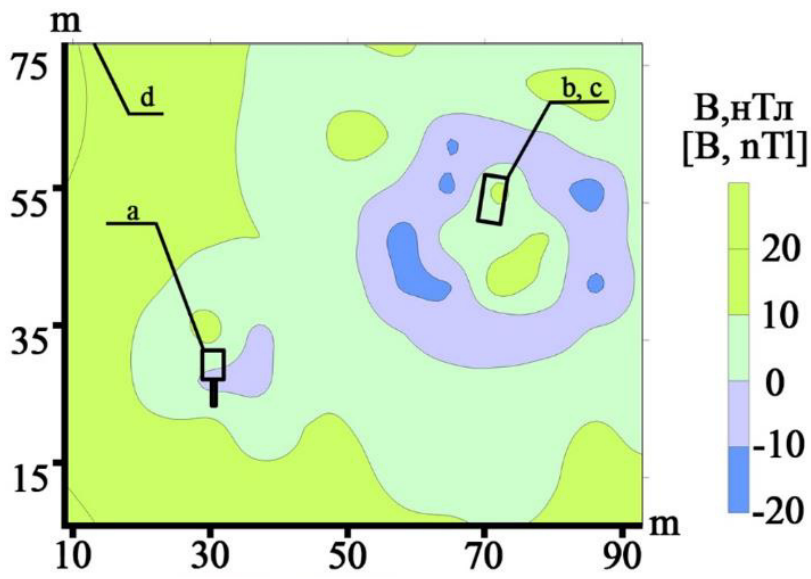

Рис. 3. Разность вычисленного и наблюдённого полей модуля вектора магнитной индукции. Пояснение: $a, b, c-$ магнитные объекты; $d$ - область магнитного моделирования.

[Fig. 3. The difference between the calculated and observed fields of the modulus of the magnetic induction vector. Explanation: $a$, $b, c$ - magnetic objects; $d$-field of magnetic modeling.]

На заключительном этапе работ были проведены прямые водолазные обследования местоположения объектов, являющихся источниками локальных магнитных аномалий, которые подтвердили наличие корпуса и башни танка, перекрытых речными наносами. На основе данных о глубинах водоёма был проведён анализ береговых склонов и выделены направления, обеспечивающие возможные варианты безопасного извлечения фрагментов танка, препятствующих судоходству и представляющих несомненную историческую ценность.
Таким образом, проведённый комплекс геофизических исследований на мелководной акватории р. Дон демонстрирует эффективность поисков техногенных объектов, залегающих на относительно небольших глубинах от уровня дна. Полученные результаты показывают высокое качество и достоверность результативной информации об искомых объектах и, кроме того, гарантируют существенную экономию финансовых затрат на геофизические работы по сравнению с традиционными методами исследований.

\section{Заключение}

Основным результатом наших исследований, направленных на обеспечение безопасности хозяйственной деятельности на мелководных акваториях, является выработка оптимального по эффективности и стоимости выполнения комплекса геофизических работ по поискам техногенных объектов. Предлагаемый комплекс инженерно-геофизических изысканий на мелководье включают последовательность работ:

1. Применение гидроакустического комплекса. Использование компактных гидролокаторов бокового обзора на мелководной акватории повысило содержательность и достоверность произведенных исследований. Гидролокация, как средство визуализации дает оценку состояния дна всей обследуемой акватории, тем самым помогает выявить объекты антропогенного или природного происхождения.

2. Применение гидромагнитной съёмки. В отсутствии гидроакустического контакта с ожидаемым объектом поиска, значимость магниторазведки резко возрастает. При выполнении полевых работ шаг наблюдений $\leq 1 / 5 H$ гарантирует выявление локальных аномалий модуля вектора индукции магнитного поля, тем самым проявляя цели антропогенного происхождения, расположенные, как на дне водной преграды, так и в донных отложениях. Стоит отметить, что в результате выполненного компьютерного моделирования было рассчитано модельное поле модуля вектора магнитной индукции, погрешность которого составила 3.9 \% по отношению к наблюденному полю, что гарантирует достоверность проведенных оценок параметров и пространственного положения намагниченных фрагментов исследуемого объекта.

3. Применение геоакустического комплекса. Задействование компактных поддонных профилографов в стесненных условиях реки, повысило надежность определения реального положения донной поверхности. Полученные данные о рельефе дна легли в основу: a) интерпретации и моделирования магнитного поля, как верхнее ограничение для оценок положения фрагментов танка; б) карты глубин, на базе которой разработан план по безопасному извлечению фрагментов танка.

Опираясь на рассмотренный пример, можно сказать, что задачи, стоящие перед предлагаемыми методами, решаются крайне успешно. Это не означает, что данный комплексный подход следует считать универ- 
сальным [4], однако решение главной задачи: локализация техногенных объектов на мелководной акватории с высокой точностью - достигается при незначительных затратах. В целом, использование данного комплекса методов является, по нашему мнению, эффективным инструментом решения технических задач контроля техногенных артефактов [13], и позволяет успешно решать инженерно-геофизические задачи обнаружения магнитных источников. Базируясь на полученных результатах можно сделать вывод о том, что, использование предложенных геофизических методов на мелководных акваториях обосновано.

Конфликт интересов: Авторы декларируют отсутствие явных и потенциальных конфликтов интересов, связанных с публикацией настоящей статьи.

\section{ЛИТЕРАТУРА}

1. Владов М. Л., Старовойтов А. В. Обзор геофизических методов исследований при решении инженерно-геологических и инженерных задач. М.: ГСД Продакшен. 1998. 67 с.

2. Манштейн А. К. Малоглубинная геофизика : Пособие по спецкурсу. Новосибирск: Изд. НГУ, 2002. 135 с.

3. Глазнев В. Н., Стариков В. С. Применение методов магнитометрической съёмки для исследования подводных переходов трубопроводов различной протяжённости в условиях мелководья // Сборник докладов 5-ой Международной научно-практической конференции «Инженерная и рудная геофизика 2009». 2009. DOI: 10.3997/2214-4609.201403827.

4. Стариков В. С. Геофизические работы при изучении техногенных объектов на мелководных акваториях // Bonросы теории и практики геологической интерпретации геофизических полей: Материалы 47-й сессии Международного научного семинара Д. Г. Успенского - В. Н. Страхова. Воронеж: «Научная книга». 2020. С. 261-265.

5. Справочник геофизика. Под ред. В. Е. Никитского, Ю. С. Глебовского. М.: Недра. 1990. 367 с.

6. Галлямов И. И., Ишемгужин Е. И. Введение в высокоточную магнитную съёмку внутрипромысловых подземных трубопроводов : Учебное пособие. Уфа : Изд-во Уфим. гос. нефтяного техн. ун-та. $2000.70 \mathrm{c}$.

7. Крапивский Е. И., Некучаев В. О. Дистанционная магнитометрия газонефтепроводов. Ухта: Изд. УГТУ. 2011. 142 с. 8. Zhao W., Huang X., Chen S., Zeng Z., Jin S. A detection system for pipeline direction based on shielded geomagnetic field // Int. J. of Pressure Vessels and Piping. 2014. V. 113. № 1. P $10-14$.

9. Агиней Р. В., Мусонов В. В., Гуськов С. С. Моделирование магнитных аномалий при проведении магнитометрического контроля трубопроводов с поверхности грунта // Tpyбопроводный транспорт. 2013. № 1. С. 40-44.

10. Загидулин Т. Р. Расчёт магнитного поля стальной трубы конечной протяжённости в постоянном однородном намаг- ничивающем поле // Контроль и диагностика. 2014. № 6. C.15-24. DOI:10.14489/td.2014.06.

11. Новикова П. Н. Ворошилов П. Н, Копытин В. В., Субботин П. А., Калашникова М. М., Темиров П. А. Инженерная магниторазведка при обнаружении подземных коммуникаций в условиях помех техногенного происхождения // 18-я молодёжная научн. школа по геофизике. Сборник научных материалов. Пермь: ГИ УрО РАН. 2017. С. 147-151.

12. Глазнев В. Н., Стариков В. С. Остаточная намагниченность и внешнее магнитное поле прямошовных стальных труб, как объекта инженерных изысканий // Вестник Воронежского государственного университета. Серия: Геология. 2018. № 3. С. 83-92.

13. Стариков В. С., Глазнев В. Н. Магнитные свойства и аномальное магнитное поле прямошовных стальных труб // Вопросы теории и практики геологической интерпретации гравитационных, магнитных и электрических полей: Сборник научных трудов. Вып. 1 (46). Пермь: ГИ УрО РАН, ПГНИУ, 2019. C. 337-340.

14. Furness, P. The magnetic fields of steel drums // Journal of Applied Geophysics. 2002. V. 51. P. 63-74.

15. Глазнев В. Н., Стариков В. С. Оценка разрешающей способности магнитного градиентометрического метода при решении инженерных геофизических задач // Материалы XVI международной конференции «Структура, свойства, динамика и минерагения литосферы Восточно-Европейской платформы». Воронеж : ИПЦ «Научная книга». 2010. С. 193-196. 16. Гершанок, Л. А. Малоглубинная магниторазведка в условиях промышленных помех // Вестник Пермского университета. Сер.: Геология. 2013. Вып. 1 (18). С. 34-49.

17. Семевский Р. Б., Аверкиев В. В., Яроцкий В. А. Специальная магнитометрия. СПб.: Наука, 2002. 228 с.

18. Стариков В. С., Кочетов М. В. Оценка разрешающей способности гидромагнитной градиентометрической съёмки // Инновационные методики геофизических исследований : Материалы ежегодной молодёжной научной конференции кафедры геофизики Воронежского государственного университета. Воронеж : ИПЦ «Научная книга». 2018. С. 90-94. 19. Глазнев В. Н. Оценка границ применимости стохастических моделей потенциальных полей // Вестник Воронежского государственного университета. Серия: Геология. 1999. № 8. C. $153-156$.

20. Булах Е. Г., Левашов С. П. Построение геоплотностных моделей методом последовательного накопления и разрастания аномальных масс // Изучение литосферы геофизическими методами. Киев : Наукова думка. 1987. С. 37-47.

21. Стариков В.С. Инженерная магнитометрия при исследовании технического состояния стальных трубопроводов большого диаметра // Вестник Воронежского государственного университета. Серия: Геология. 2016. № 3. С. 114- 118. 22. Глазнев В.Н., Лошаков Г. Г. Об одном методе моделирования рудных объектов с использованием адаптивной аппроксимации // Вестник Воронежского государственного университета. Серия: Геология. 2012, № 1. С. 243-246. 
UDC 550.838.22

ISSN 1609-0691

DOI: https://doi.org/10.17308/geology.2021.1/3339

Received: 08.02.2021

Accepted: 01.03.2021

Published online: 31.03 .2021

\title{
Methods of geophysics engineering in the search for technogenic structures in shallow waters
}

\author{
CC2021 V.S. Starikov ${ }^{凶}$ \\ «PETER» JOINT - STOCK C ${ }^{\circ}$. Diving Services. \\ 2, bld.A, st. Ostuzheva, Voronezh 394042, Russian Federation
}

\begin{abstract}
:
Introduction: In the present work we studied the problem of the identification of technogenic structures in shallow waters which were concealed due to the process of sedimentation. To solve the task, we suggested a complex approach based on the results of the interpretation of the conducted measurements of the modulus of the magnetic induction vector in accordance with the proposed survey method supported by seismoacoustics methods.

Methodology: Unwieldy equipment and large water vessels cannot be used in shallow waters or in an area limited by coastlines. Thus, it was suggested to develop a set of methods and a working methodology involving the use of small water vessels during the search and discovery of unpredictable discrete magnetised structures. Based on the previous studies involving extensive linear technogenic structures, we used hydroacoustic, magnetometric, and geoacoustic methods in a step-by-step manner. Methodological provision of each type of the conducted work includes solvability which guarantees the identification of the goals and sufficiency of the collected information providing a general understanding of the condition of the water area.

Results and discussion: A hydroacoustic survey, as a visualisation tool, allowed studying the degree of the bottom's cluttering with anthropogenic structures, although it could not unambiguously identify the fragments of the tank, as over a long period of time it had been all levelled with the stream sediment. Two significant anomalies of the magnetic field were found as a result of the conducted magnetometric observations. A quantitative interpretation of magnetic anomalies was conducted for a two-dimensional case: a conversion to the upper half area was performed, and an estimate of the depth of the structures was presented. As a result of the conducted work using the geoacoustic method, the depths of the river's water area were specified. The overall results of the used set of methods allowed solving the direct problem of magnetometry using Mathcad 14. As a result of the modelling performed in Mathcad 14, the calculated field of the modulus of the induction vector from the set of model sources showed good correlation with the observed field. Direct diving surveys which were performed after the completion of the survey stage confirmed the presence of the body of the tank and tank tower covered with river sediments.

Conclusions: The main results of our research aimed at ensuring the safety of economic activities in shallow water areas while searching for unpredictable magnetised technogenic structures are the improvement of the hydromagnetic survey method with the further interpretation of data, including computer modelling, as well as the development of an optimal complex of geophysical works.
\end{abstract}

Keywords: shallow water, technogenic structure, set of geophysical methods, magnetic anomalies, magnetic field modelling.

For citation: Starikov V. S. Methods of geophysics engineering in the search for technogenic structures in shallow waters. Vestnik Voronezhskogo gosudarstvennogo universiteta. Seriya: Geologiya - Proceedings of Voronezh State University. Series: Geology, 2021, no. 1, pp. 75-81. DOI: https://doi.org/10.17308/geology.2021.1/3339

Conflict of interests: The authors declare the absence of obvious and potential conflicts of interest related to the publication of this article.

The content is available under Creative Commons Attribution 4.0 License.

$\bowtie$ Vadim A. Starikov, e-mail: stvase@mail.ru 


\section{REFERENCES}

1. Vladov M. L., Starovoitov A. V. Obzor geofizicheskikh metodov issledovanii pri reshenii inzhenerno-geologicheskikh $i$ inzhenernykh zadach [Review of geophysical research methods in solving engineeringgeological and engineering problems]. Moscow, GSD Prodakshen publ., 1998. 67 p. (In Russ)

2. Manshtein A. K. Maloglubinnaya geofizika : Posobie po spetskursu [Shallow Geophysics: A Specialized Course Manual]. Novosibirsk, NGU publ, 2002. 135 p. (In Russ)

3. Glaznev V. N., Starikov V. S. Primenenie metodov magnitometricheskoi s"emki dlya issledovaniya podvodnykh perekhodov truboprovodov razlichnoi protyazhennosti $\mathrm{v}$ usloviyakh melkovod'ya [Application of magnetometric methods for studying underwater pipelines of various lengths in shallow water conditions]. Sbornik dokladov 5-oi Mezhdunarodnoi nauchno-prakticheskoi konferentsii «Inzhenernaya i rudnaya geofizika 2009» [Collection of reports of the 5th International Scientific and Practical Conference "Engineering and Ore Geophysics 2009"]. 2009 DOI: 10.3997/2214-4609.201403827. (In Russ)

4. Starikov V.S. Geofizicheskie raboty pri izuchenii tekhnogennykh ob"ektov na melkovodnykh akvatoriyakh [Geophysical work in the study of technogenic objects in shallow water areas]. Voprosy teorii i praktiki geologicheskoi interpretatsii geofizicheskikh polei: Materialy 47-i sessi Mezhdunarodnogo nauchnogo seminara D. G. Uspenskogo - V. N. Strakhova [Questions of theory and practice of geological interpretation of geophysical fields: Proceedings of the 47th session of the Internationa Scientific Seminar of D.G. Uspensky, V. N. Strakhova], Voronezh, Nauchnaya kniga publ., 2020, pp. 261-265. (In Russ)

5. Spravochnik geofizika [Geophysicist Handbook]. Ed.. V.E. Nikitskogo, Yu. S. Glebovskogo. Moscow, Nedra publ., 1990. 367 p. (In Russ) 6. Gallyamov I. I., Ishemguzhin E. I. Vvedenie v vysokotochnuyu magnitnuyu s"emku vnutripromyslovykh podzemnykh truboprovodov. Uchebnoe posobie [Vvedenie v vysokotochnuyu magnitnuyu shooting vnutripromyslovykh podzemnykh truboprovodov: Uchebnoe posobie]. Ufa, Ufim. gos. neftyanogo tekhn. un-ta publ. 2000. 70 p. (In Russ)

7. Krapivskii E. I., Nekuchaev V. O. Distantsionnaya magnitometriya gazonefteprovodov [Remote magnetometry of gas and oil pipelines]. Ukhta, UGTU publ., 2011. 142 p. (In Russ)

8. Zhao W., Huang X., Chen S., Zeng Z., Jin S. A detection system for pipeline direction based on shielded geomagnetic field. Int. J. of Pressure Vessels and Piping, 2014, vol. 113, no 1, pp. 10-14.

9. Aginei R. V., Musonov V. V., Gus'kov S. S. Modelirovanie magnitnykh anomalii pri provedenii magnitometricheskogo kontrolya truboprovodov s poverkhnosti grunta [Modeling of magnetic anomalies during magnetometric inspection of pipelines from the soil surface]. Truboprovod nyi transport-Pipeline transport, 2013, no. 1, pp. 40-44. (In Russ)

10. Zagidulin T. R. Raschet magnitnogo polya stal'noi truby konechno protyazhennosti $\mathrm{v}$ postoyannom odnorodnom namagnichivayushchem pole [Calculation of the magnetic field of a steel pipe of finite length in a constant uniform magnetizing field]. Kontrol' i diagnostika-Monitoring and diagnostics, 2014, no 6, pp.15-24. (In Russ) DOI:10.14489/td.2014.06.

11. Novikova P. N. Voroshilov P. N, Kopytin V. V., Subbotin P. A., Kalashnikova M. M., Temirov P. A. Inzhenernaya magnitorazvedka pri obnaruzhenii podzemnykh kommunikatsii v usloviyakh pomekh tekhnogennogo proiskhozhdeniya [Engineering magnetic prospecting for detecting underground utilities in conditions of technogenic interference]. 18-ya molodezhnaya nauchn. shkola po geofizike. Sbornik nauchnykh materialov [18th youth scientific. school of geophysics. Collection of scientific materials]. Perm, GI URO RAN, 2017, pp. 147-151. (In Russ) 12. Glaznev V. N., Starikov V.S. Ostatochnaya namagnichennost' i vneshnee magnitnoe pole pryamoshovnykh stal'nykh trub, kak ob"ekta inzhenernykh izyskanii [Residual magnetization and external magnetic field of longitudinal welded steel pipes as an object of engineering survey]. Vestnik Voronezhskogo gosudarstvennogo universiteta. Seriya: Geologiya $=$ Proceedings of Voronezh State University. Series: Geology, 2018, no. 3, pp. 83-92. (In Russ)

13. Starikov V. S., Glaznev V. N. Magnitnye svoistva i anomal'noe magnitnoe pole pryamoshovnykh stal'nykh trub [Magnetic properties and anomalous magnetic field of longitudinal welded steel pipes]. Voprosy teorii i praktiki geologicheskoi interpretatsii gravitatsionnykh, magnitnykh i ehlektricheskikh polei: Sbornik nauchnykh trudov [Questions of theory and practice of geological interpretation of gravitational, magnetic and electric fields: Collection of scientific papers.]. Vol. 1 (46). Perm, G URO RAN, PGNIU, 2019, pp. 337-340. (In Russ)

14. Furness P. The magnetic fields of steel drums. Journal of Applied Geophysics, 2002, vol. 51, pp. 63-74.

15. Glaznev V. N., Starikov V. S. Otsenka razreshayushchei sposobnosti magnitnogo gradientometricheskogo metoda pri reshenii inzhenernykh geofizicheskikh zadach [Estimation of the resolving power of the magnetic gradiometer method in solving engineering geophysical problems]. Materialy XVI mezhdunarodnoi konferentsii «Struktura, svoistva, dinamika i minerageniva litosfery Vostochno-Evropeiskoi platformy» [Materials of the XVI International Conference "Structure, Properties, Dynamics and Minerageny of the Lithosphere of the East European Platform"]. Voronezh, IPTS Nauchnaya kniga publ., 2010, pp. 193-196. (In Russ) 16. Gershanok L. A. Maloglubinnaya magnitorazvedka v usloviyakh promyshlennykh pomekh [Shallow magnetic prospecting in industrial noise conditions]. Vestnik Permskogo universiteta. Ser.: Geologiya - Bulletin of Perm University. Geology, 2013, vol. 1 (18), pp. 34-49. (In Russ) 17. Semevskii R. B., Averkiev V. V., Yarotskii V. A. Spetsial'naya magnitometriya [Special magnetometry]. Saint Petersburg, Nauka publ., 2002. 228 p. (In Russ)

18. Starikov V. S., Kochetov M. V. Otsenka razreshayushchei sposobnost gidromagnitnoi gradientometricheskoi s"emki [Estimation of the resolution of the hydromagnetic gradient metric survey]. Innovatsionnye metodiki geofizicheskikh issledovanii : Materialy ezhegodnoi molodezhnoi nauchnoi konferentsii kafedry geofiziki Voronezhskogo gosudarstvennogo universiteta [Innovative methods of geophysical research: Materials of the annual youth scientific conference of the Department of Geophysics, Voronezh State University]. Voronezh, Nauchnaya kniga publ,. 2018, pp. 90-94. (In Russ)

19. Glaznev V. N. Otsenka granits primenimosti stokhasticheskikh modelei potentsial'nykh polei [Estimation of the limits of applicability of stochastic models of potential fields]. Vestnik Voronezhskogo gosudarstvennogo universiteta. Seriya: Geologiya = Proceedings of Voronezh State University. Series: Geology, 1999, no 8, pp. 153-156. (In Russ)

20. Bulakh E .G., Levashov S. P. Postroenie geoplotnostnykh modelei metodom posledovatel'nogo nakopleniya i razrastaniya anomal'nykh mass [Construction of geo-density models by the method of sequential accumulation and growth of anomalous masses]. Izuchenie litosfery geofizicheskimi metodami [Study of the lithosphere by geophysical methods]. Kiev, Naukova dumka publ., 1987, pp. 37-47. (In Russ)

21. Starikov V. S. Inzhenernaya magnitometriya pri issledovanii tekhnicheskogo sostoyaniya stal'nykh truboprovodov bol'shogo diametra [Engineering magnetometry in the study of the technical condition of large-diameter steel pipelines]. Vestnik Voronezhskogo gosudarstvennogo universiteta. Seriya: Geologiya = Proceedings of Voronezh State University. Series: Geology, 2016, no. 3, pp. 114-118. (In Russ)

22. Glaznev V. N., Loshakov G. G. Ob odnom metode modelirovaniya rudnykh ob"ektov s ispol'zovaniem adaptivnoi approksimatsii [On one method for modeling ore objects using adaptive approximation]. Vestnik Voronezhskogo gosudarstvennogo universiteta. Seriya: Geologiya = Proceedings of Voronezh State University. Series: Geology, 2012, no. 1, pp. 243-246. (In Russ)

Vadim S. Starikov - engineer-geophysicist, «PETER» JOINT STOCK $\mathrm{C}^{\circ}$. Diving Services, Voronezh, Russian Federation e-mail: stvase@mail.ru; ORCID http://orcid.org/ 0000-0002-2071-9835.

The author have read and approved the final manuscript. 